\title{
Platform Incubator with Movable XY Stage: A New Platform for Implementing In-Cell Fast Photochemical Oxidation of Proteins
}

\author{
Danté Johnson ${ }^{1}$, Benjamin Punshon-Smith ${ }^{2}$, Jessica A. Espino ${ }^{1}$, Anne Gershenson ${ }^{3}$, Lisa M. Jones ${ }^{1}$ \\ ${ }^{1}$ Department of Pharmaceutical Sciences, University of Maryland Baltimore ${ }^{2}$ Technology Research Center, University of Maryland Baltimore \\ County ${ }^{3}$ Department of Biochemistry and Molecular Biology, University of Massachusetts
}

\section{Corresponding Author}

Lisa M. Jones

ljones@rx.umaryland.edu

\section{Citation}

Johnson, D., Punshon-Smith, B., Espino, J.A., Gershenson, A., Jones, L.M. Platform Incubator with Movable XY Stage: A New Platform for Implementing In-Cell Fast Photochemical Oxidation of Proteins. J. Vis. Exp. (171), e62153, doi:10.3791/62153 (2021).

\section{Date Published}

May 17, 2021

DOI

$10.3791 / 62153$

URL

jove.com/video/62153

\section{Abstract}

Fast Photochemical Oxidation of proteins (FPOP) coupled with mass spectrometry (MS) has become an invaluable tool in structural proteomics to interrogate protein interactions, structure, and protein conformational dynamics as a function of solvent accessibility. In recent years, the scope of FPOP, a hydroxyl radical protein foot printing (HRPF) technique, has been expanded to protein labeling in live cell cultures, providing the means to study protein interactions in the convoluted cellular environment. In-cell protein modifications can provide insight into ligand induced structural changes or conformational changes accompanying protein complex formation, all within the cellular context. Protein footprinting has been accomplished employing a customary flow-based system and a $248 \mathrm{~nm} \mathrm{KrF}$ excimer laser to yield hydroxyl radicals via photolysis of hydrogen peroxide, requiring 20 minutes of analysis for one cell sample.To facilitate time-resolved FPOP experiments, the use of a new 6-well plate-based IC-FPOP platform was pioneered. In the current system, a single laser pulse irradiates one entire well, which truncates the FPOP experimental time frame resulting in 20 seconds of analysis time, a 60 -fold decrease. This greatly reduced analysis time makes it possible to research cellular mechanisms such as biochemical signaling cascades, protein folding, and differential experiments (i.e., drug-free vs. drug bound) in a time-dependent manner. This new instrumentation, entitled Platform Incubator with Movable XY Stage (PIXY), allows the user to perform cell culture and IC-FPOP directly on the optical bench using a platform incubator with temperature, $\mathrm{CO}_{2}$ and humidity control. The platform also includes a positioning stage, peristaltic pumps, and mirror optics for laser beam guidance. IC-FPOP conditions such as optics configuration, flow rates, transient transfections, and $\mathrm{H}_{2} \mathrm{O}_{2}$ concentration in 
PIXY have been optimized and peer-reviewed. Automation of all components of the system will reduce human manipulation and increase throughput.

\section{Introduction}

Protein footprinting techniques can reveal profound information on the organization of proteins. These essential structural biology MS-based techniques are a component of the mass spectrometry toolbox. These methods probe protein higher order structure (HOS) and synergy via covalent labeling ${ }^{1,2,3,4}$. Fast photochemical oxidation of proteins (FPOP) employs hydroxyl radicals to oxidatively modify solvent accessible side chains of amino acids ${ }^{5,6}$ (Table 1). The method utilizes an excimer laser at $248 \mathrm{~nm}$ for photolysis of hydrogen peroxide $\left(\mathrm{H}_{2} \mathrm{O}_{2}\right)$ to generate hydroxyl radicals. Theoretically, 19 of the 20 amino acids can be oxidatively modified with Gly being the lone exception. However, owing to the varying reactivity rates of amino acids with hydroxyl radicals, modification of only a subset of these has been observed experimentally. Still, the method does have the potential for analysis over the length of a protein sequence $^{5}$. FPOP modifies proteins on the microsecond timescale, making it useful in studying weak interactions with fast off rates. Solvent accessibility changes upon ligandbinding or a change in protein conformation, thus, the power of the method lies in the comparison of the labeling pattern of a protein in multiple states (i.e., ligand-free compared to ligand-bound). As a result, FPOP has been successful in identifying protein-protein and protein-ligand interaction sites and regions of conformational change $\mathrm{e}^{7,8,9,10}$. The FPOP method has been extended from the study of purified protein systems to in-cell analysis. In-cell FPOP (IC-FPOP) can oxidatively modify over a thousand proteins in cells to provide structural information across the proteome ${ }^{11,12}$. The conventional IC-FPOP platform utilizes a flow system to flow cells single file past the laser beam. The development of this system allowed individual cells to have equal exposure to laser irradiation. This led to a 13-fold rise in the number of oxidatively labeled proteins ${ }^{12}$. However, a limitation of the flow system is the length of a single sample experiment consisting of a 10-minute irradiation interval during which modification takes place and an additional 10-minute wash cycle. The time constraints of IC-FPOP makes it unsuitable for studying short lived protein folding intermediates or changes that exist among interaction networks in biochemical signaling cascades. This temporal limitation inspired the design of a new IC-FPOP platform equipped with higher throughput.

To accurately measure protein higher order structure in the native cell environment, the new design allows cell culture to be accomplished directly at the laser platform, which enables IC-FPOP to be high throughput. This setup also allows minimized perturbations to the cellular environment, in contrast to IC-FPOP using flow where adherent cells must be removed from the substrate. The new platform permits IC-FPOP to occur in a sterile incubation system using a $\mathrm{CO}_{2}$ and temperature-controlled stage top chamber while utilizing configured mirror optics for laser beam guidance, a positioning system for $X Y$ motion, and peristaltic pumps for chemical exchange. The new platform for conducting ICFPOP is entitled Platform Incubator with Movable XY Stage (PIXY) (Figure 1). In PIXY, IC-FPOP is carried out on human cells grown in six-well plates within the platform incubator chamber. For this configuration, the laser beam is reflected downward onto the plate using beam compatible mirrors as 
a positioning stage that holds the incubator is moved, in the $\mathrm{XY}$-plane, so the laser beam is strategically aligned to only irradiate one well at a time. Validation studies show that IC-FPOP can be performed faster in PIXY than in the flow system and leads to increased amino acid modifications per protein. The development of this new IC-FPOP platform will expound upon the knowledge that can be gained from cellular experiments $^{13}$.

\section{Protocol}

\section{Assembly of Platform Incubator with Movable $\mathrm{XY}$ stage}

NOTE: The new platform includes the incubation system, the positioning stage and controllers, the peristaltic pumps, the $248 \mathrm{~nm} \mathrm{KrF}$ excimer laser, and the optical mirrors assembled on an Imperial optical breadboard.

1. Assemble the incubation system: the temperature unit, carbon dioxide unit, humidifier, air pump, and touch monitoring system (Figure 2A-E).

NOTE: Detailed assembly instructions are provided by the manufacturer. The incubation system must stabilize to $5 \% \mathrm{CO}_{2}, 37^{\circ} \mathrm{C}$, and $85 \%$ humidity before growing cells within the incubator. These parameters may depend on the cell line.

2. Assemble the custom six-well plate incubator, nanopositioning drive stage, and $X Y$ drive stage. The former screws into the latter, respectively.

NOTE: Detailed assembly instructions provided by the manufacturer.

3. Connect the four peristaltic pumps in a "daisy chain" sequence via RS-232 cables, with an RS-232 to USB cable connected to the controlling computer. Connect
$3.18 \mathrm{~mm}$ ID polymer tubing (e.g., Tygon) to every channel on each pump channel roller.

NOTE: Each pump has four rollers. Direction and flow rate of rollers are manually manipulated.

4. Insert $1 / 16 " \times 1 / 8$ " connectors at the end of each $3.18 \mathrm{~mm}$ ID polymer tube. Insert the $1 / 16$ " end of the connector into 1.59 ID polymer tubing, then insert the polymer tubing into the incubator via custom ports. Place the other end of the tube in the solution that will be infused during ICFPOP experimentation.

NOTE: For the perfusion lines, the incubator has 36 custom ports for tubing lines all around the periphery to accommodate all the reagents used.

5. Screw one $50 \mathrm{~mm}, 248 \mathrm{~nm}, 45^{\circ}$ excimer laser line mirror within a kinematic mirror mount for $\varnothing 2 "$ optics into the breadboard 10-11 grid points from the $248 \mathrm{~nm}$ laser aperture. Place the second mirror at a $90^{\circ}$ angle to the first on the other side of the incubator. Angle the second mirror downward at approximately $45^{\circ}$ for laser beam guidance to the incubator (Figure 2F-J).

\section{Synchronization and initial automation of system via integration software}

1. Install the latest version of the integration software needed to control the drivers and pumps.

2. Referring to the pump manual, rename the pumps starting with ' 5 ' and increasing in value. The commands can be sent to the pump system using the Manual Control sub-program in the integration software.

NOTE: Setting a pump to Channel Mode automatically changes the pump naming convention into the set, 1,2 , 3 and 4 , corresponding to the four pump channels.

3. Open the integration software program for the automation of the platform incubator. 
4. Choose the appropriate USB comport device name (e.g., COM4) corresponding to the pump system USB cable from the connection dropdown menu labelled Comport for pumps.

5. Click the Script Builder button to edit/create a script for the automated platform incubator platform. Click Save Script to save the sequence as a text file (Figure 3A). NOTE: The script builder has a user interface for defining the script with definitions of pump number, direction, rate, volume, tubing diameter, channel mode, and timed delay.

6. If channel mode is desired in the making of the script, a step in the script must be dedicated to changing the pump in and out of channel mode, and the following steps corresponding to the pump channels must be labelled as pumps 1-4.

NOTE: This ensures that no two pumps can simultaneously be in channel mode during a platform incubator script and that each pump is switched back to legacy mode after the commands have been sent to the needed channels.

7. Click the Read Script button and choose the appropriate script file desired for the platform incubator operation.

8. Switch the Run Sequence button to the ON position (green) to run the script and then click the START button

(Figure 3B) .

\section{Grow cells in the platform incubator}

NOTE: Cells must be placed in the platform incubator under sterile conditions in a cell culture hood the day before experimentation.

1. Unscrew the platform incubator from the nanopositioning stage and disconnect the temperature, gas, and humidifier lines. After spraying the incubator with $70 \%$ ethanol, place it in a cell culture hood.

2. Grow cells in a $\mathrm{T}-175$ to about $80-90 \%$ confluency prior to transfer.

NOTE: The term confluency is used as a measure of the number/coverage of the cells in a cell culture dish or a flask.

3. Remove media and rinse with buffer.

4. Detach cells using trypsin-EDTA using manufacturer's protocol.

5. Resuspend in $8 \mathrm{~mL}$ of media and count the cells.

6. Seed fibronectin/collagen ${ }^{14}$ coated six-well plates with approximately 90-95 $\mu \mathrm{L}$ of resuspended cells in each well. This volume will be appropriate to achieve $80-90 \%$ confluency $(\sim 1.2$ million cells) in each well on the next day.

NOTE: Six-well plates must be coated with collagen prior to seeding. The reagents used during IC-FPOP are infused with fast flow rates. Coated plates ensure cells are not prematurely detached due to infusion of reagents.

7. Place the seeded plate into the platform incubator and cover with the quartz lid.

NOTE: During design and development, a glass incubator lid was changed to quartz, so it is compatible with the ultraviolet laser light.

8. Replace and secure the platform incubator back on the nanopositioning drive stage. Reconnect the temperature, gas, and humidifier lines.

9. Let cells grow to confluency overnight.

NOTE: The following cell culture steps are optional and are intended for experiments in which human cells are 
transiently transfected. These steps assess transfection efficiency under cell culture conditions.

10. Transfect the plasmid containing the gene for the chimeric protein GCaMP2 into HEK 293 cells using a commercial cationic-lipid transfection kit.

11. Perform transient transfections of GCaMP2 in HEK293T cells in two six-well plates.

12. Incubate one six-well plate in the platform incubator, and the second six-well plate in a standard $\mathrm{CO}_{2}$ incubator.

13. Compare transfection efficiency within each plate by fluorescence imaging using a fluorescence microscope.

\section{Make quench buffer and $\mathrm{H}_{2} \mathrm{O}_{2}$}

1. Make $100 \mathrm{~mL}$ of quench buffer containing $125 \mathrm{mM}$ N-tert-Butyl-a-phenylnitrone (PBN) and $125 \mathrm{mM} \mathrm{N}, \mathrm{N}^{\prime}-$ Dimethylthiourea (DMTU).

NOTE: DMTU and PBN are free radical scavengers and are cell permeable.

2. Dilute $\mathrm{H}_{2} \mathrm{O}_{2}$ to $200 \mathrm{mM}$. Each sample requires $6 \mathrm{~mL}$ of $\mathrm{H}_{2} \mathrm{O}_{2}$ to fully immerse the layer of cells at the bottom of each well.

NOTE: The quench buffer is made the day before and stored at $4{ }^{\circ} \mathrm{C}$ overnight, protected from light. Dilute the $\mathrm{H}_{2} \mathrm{O}_{2}$ on the day of the experiment.

\section{Set up the platform incubator for IC-FPOP}

NOTE: The platform incubator must be assembled under sterile conditions. Assemble the incubator in a sterile cell culture hood.

1. Unscrew the platform incubator from the positioning stage and disconnect the temperature, gas, and humidifier lines.
2. After spraying the incubator with $70 \%$ ethanol, place it in the cell culture hood.

3. Remove the six-well plate from the platform incubator, secure the plate's original lid, and confirm cell confluency using a microscope.

4. After cell confluency has been confirmed, replace the six-well plate with confluent cells back in the platform incubator inside the cell culture hood.

5. Insert three precut $(15 \mathrm{~cm}) 1.59 \mathrm{ID}$ polymer tubes in each well via the embedded ports. Flush the tubes to the well walls and hold with custom 3D printed rings.

NOTE: Six $33 \mathrm{~mm}$ PLA filament 3D printed rings were custom designed to secure the tubing to the walls of the plate without disturbing the cells or getting in the way of the laser pulse.

6. Cover the platform incubator with its quartz lid. Replace and secure the stage top incubator on the positioning system. Reconnect the temperature, gas, and humidifier lines.

7. Connect 1.59 ID polymer tubing to $1 / 16$ " end of the $1 / 16 \times 1 / 8$ " connectors.

\section{Performing IC-FPOP in the platform incubator}

1. Prepare an integration software script for pump withdrawal. Use one peristaltic pump (Pump 8) to completely remove cell media from all six wells.

2. Prime solvents in each channel of the other three peristaltic pumps (Pumps 5-7). Infuse $\mathrm{H}_{2} \mathrm{O}_{2}$ and quench buffer in their respective alternating tubes until the reagents reach the incubator ports.

3. Confirm that the laser beam has been angled correctly by the mirrors and reaches the incubator uninhibited. 
NOTE: Laser safety goggles must be worn whenever the laser is in use. Do not prematurely irradiate the cells during the angling/alignment process. Use cardboard to completely cover the quartz lid when aligning the beam. Also, use a printed outline on white paper of a six-well plate to further confirm the laser beam is hitting the center of each well. Use the Continuous pulse setting at the lowest frequency and energy for alignment.

4. Check laser energy using an external sensor. Use one single laser pulse of $160 \mathrm{~mJ}$ at $50 \mathrm{~Hz}$ and $27 \mathrm{kV}$.

NOTE: A timer is needed for the following steps.

5. Prepare the integration software script for pump infusion after confirming beam alignment.

6. Begin the timer and press the Start button on the integration software pump script at the same time.

7. Infuse $200 \mathrm{mM} \mathrm{H}_{2} \mathrm{O}_{2}$ at $35 \mathrm{~mL} / \mathrm{min}$ into the first well (6-10 second mark on timer).

NOTE: There is a five second delay before a pump begins to infuse. It also takes the laser seven seconds before the pulse is triggered. The laser pulse must come immediately after $\mathrm{H}_{2} \mathrm{O}_{2}$ infusion.

8. Press the Start button on the laser software at the 5 second mark to trigger the pulse at the 11 second mark on the timer.

9. Infuse $125 \mathrm{mM}$ quench solution at $35 \mathrm{~mL} / \mathrm{min}$ into the first well immediately after the laser pulse. Manually move the positioning stage to align the next well with the laser beam.

10. Repeat the above steps 6.5-6.9 until each well has been processed.

NOTE: IC-POP is performed in technical triplicate of three laser and three non-laser samples. One processed six-well plate serves as one biological replicate.
11. In a cell culture hood, use a cell scraper to transfer the cells from each well into individual $15 \mathrm{~mL}$ conical tubes. Centrifuge cells at $1,200 \times \mathrm{g}$ for 5 minutes.

12. Discard the supernatant and resuspend cells in $100 \mu \mathrm{L}$ of RIPA lysis buffer.

13. Transfer cells to individual $1.2 \mathrm{~mL}$ polypropylene tubes.

14. Flash freeze all samples in liquid nitrogen and place in a $-80{ }^{\circ} \mathrm{C}$ freezer until use.

NOTE: The protocol can be paused here.

\section{Protein extraction, purification, and proteolysis}

1. Thaw the samples and heat at $95{ }^{\circ} \mathrm{C}$ in a heat block for 5 minutes.

2. After heating, cool on ice for 5 minutes.

3. Add 25 units of nuclease to the cell lysate to degrade single-stranded, double-stranded, linear and circular DNA and RNA and incubate at room temperate for 15 minutes.

4. Centrifuge samples at $16,000 \times \mathrm{g}$ for 10 minutes at $4{ }^{\circ} \mathrm{C}$.

5. Collect the supernatant and transfer to a clean polypropylene tube.

6. Check the protein concentration using a colorimetric protein assay.

7. Transfer $100 \mu \mathrm{g}$ of sample to a clean polypropylene tube and bring to $100 \mu \mathrm{L}$ with cell lysis buffer.

8. Reduce samples with $10 \mathrm{mM}$ dithiothreitol (DTT) at $50^{\circ} \mathrm{C}$ for 45 minutes.

9. Cool samples at room temperature for 10 minutes.

10. Alkylate with $50 \mathrm{mM}$ iodoacetamide (IAA) at room temperature for 20 minutes.

NOTE: Protect IAA from light 
11. Add $460 \mu \mathrm{L}$ of pre-chilled $\left(-20^{\circ} \mathrm{C}\right)$ acetone. Vortex samples and place at $-20^{\circ} \mathrm{C}$ overnight.

NOTE: The protocol can be paused here.

12. Next day, centrifuge samples at $16,000 \times \mathrm{g}$ for 10 minutes at $4{ }^{\circ} \mathrm{C}$.

13. Remove acetone without disrupting the protein pellet.

14. Add $50 \mu \mathrm{L}$ of $90 \%$ pre-chilled $\left(-20{ }^{\circ} \mathrm{C}\right)$ acetone. Vortex samples to mix and centrifuge at $16,000 \times \mathrm{g}$ for an additional 5 minutes at $4{ }^{\circ} \mathrm{C}$.

15. Remove acetone and let samples dry for 2-3 minutes.

16. Resuspend protein pellet with $10 \mathrm{mM}$ Tris buffer $\mathrm{pH} 8$.

17. Resuspend MS grade trypsin (20 $\mu \mathrm{g}$ stock) in $40 \mu \mathrm{L}$ of $10 \mathrm{mM}$ Tris buffer $\mathrm{pH} 8$ and add $2.5 \mu \mathrm{g}$ of trypsin to each sample.

18. Incubate samples at $37^{\circ} \mathrm{C}$ overnight.

NOTE: The protocol can be paused here.

19. Assess peptide concentration using a colorimetric peptide assay.

20. Quench the samples with $5 \%$ formic acid.

21. Transfer $10 \mu \mathrm{g}$ of sample to a clean polypropylene tube.

22. Dry the sample using a vacuum centrifuge and resuspend with $20 \mu \mathrm{L}$ of MS grade $0.1 \%$ formic acid (FA) in water.

23. Transfer each sample to clean autosampler vials with pre-slit caps.

\section{High performance Liquid Chromatography- Tandem Mass Spectrometry (LC-MS/MS)}

1. To localize FPOP modifications, analyze the digested cell lysate using LC-MS/MS analysis.
2. Use mobile phases of $0.1 \% \mathrm{FA}$ in water $(\mathrm{A})$ and $0.1 \% \mathrm{FA}$ in acetonitrile (ACN) (B).

3. Load $0.5 \mu \mathrm{g}$ of sample onto a $180 \mu \mathrm{m} \times 20 \mathrm{~mm}$ C18 (5 $\mu \mathrm{m}$ and $100 \AA$ ) trapping column and wash the column with $99 \%(A)$ and $1 \%(B)$ for 15 minutes.

4. Using a $75 \mu \mathrm{m} \times 30 \mathrm{~cm} \mathrm{C18}(5 \mu \mathrm{m}$ and $125 \AA)$ analytical column, elute and separate digested peptides with a flow rate of $0.300 \mu \mathrm{L} / \mathrm{min}$ for 120 minutes.

5. Run LC gradient as follows: $0-1$ min, $3 \%$ solvent B; 2-100 $\min , 10-45 \% \mathrm{~B} ; 100-110 \min , 45-100 \% \mathrm{~B}$; 110-115 $\min , 100 \% \mathrm{~B}$.

6. Recondition the column at 3\% (B) from 115-116 minutes and hold at 3\% (B) from 116-120 minutes.

7. Analyze eluted peptides in positive ion mode with nano electrospray ionization.

8. Acquire MS1 spectra over a m/z scan range of $375-1500$ at a resolution of 60,000

9. Set the automatic gain control (AGC) target to $5.0 e^{5}$ with a maximum injection time of $50 \mathrm{~ms}$ and $5.0 \mathrm{e}^{4}$ intensity threshold.

10. Isolate precursor ions with charge states 2-6 via data dependent acquisition (DDA) with an isolation window of $1.2 \mathrm{~m} / \mathrm{z}$ and a cycle time of 4 seconds.

11. Subject MS2 ions to high-energy collisional dissociation (HCD) (32\% normalized energy).

12. Exclude peptides after 1 MS/MS acquisition for 60 seconds.

13. Set MS/MS resolution to 15,000 with an $\mathrm{AGC}$ target of $5.0 \mathrm{e}^{4}$ and a maximum injection time of $35 \mathrm{~ms}$. 


\section{Proteome discoverer/data processing}

1. Search tandem raw data files on available bottom-up proteomics analysis software against a relevant Homo sapiens protein database and digest enzyme.

2. Set the protein analysis search parameters.

3. Set fragment tolerance to $0.02 \mathrm{Da}$ and parent ion tolerance to $10 \mathrm{ppm}$.

4. Set enzyme specificity to trypsin and allow for one missed cleavage.

5. Set mass range to $375-1500 \mathrm{~m} / \mathrm{z}$.

6. Establish peptide confidence at $95 \%$ (medium) and residue confidence at $99 \%$ (high).

7. Accept proteins if at least two distinct peptides are identified with the $5 \%$ discovery rate (FDR) filter.

8. Set carbamidomethylation as a static modification and all known hydroxyl radical side-chain modifications ${ }^{15,16}$ as dynamic modifications.

9. Once files are finished searching, export sequence, modification locations, protein accession, spectrum file, precursor abundance, and retention time information to an electronic database.

10. Calculate the extent of modification per peptide or residue from this equation:

$\underline{\Sigma E I C}$ area modified

$\Sigma E I C$ area

NOTE: EIC area modified is the extracted ion chromatographic area (EIC) of the peptide or residue with an oxidative modification, and EIC area is the total area of the same peptide or residue with and without the oxidative modification. Over time, protein in the presence of hydrogen peroxide will oxidize, resulting in background oxidations. To calculate the extent of modification, the area of the modified peptide is divided by the total area. A non-irradiated control sample accounts for background oxidation. The background oxidation from the control sample is subtracted out from the laser treated sample to identify an FPOP modification.

\section{Representative Results}

To confirm that the platform incubator conditions are sufficient for cell culture at the laser platform, GCaMP2 was transiently transfected into HEK293T and transfection efficiency for both plates was assessed via fluorescence imaging (Figure 4A). GCaMP2 is a calcium sensing fluorescent protein used as a genetically encoded intracellular calcium indicator. It is a fusion of green fluorescent protein (GFP) and the calcium-binding protein, calmodulin. A luciferase assay was performed on HEK 293 cells transfected with plasmid prlTK in order to quantify transfection efficiency (Figure 4B). These results show that the platform incubator exceeded the performance of the standard incubator, with a 1.13-fold increase in transfection efficiency, providing a quantitative benchmark for optimal cell culture environment.

FPOP modifications in HEK293T cells labeled in the flow system were compared to those labeled in the platform incubator and showed that the platform incubator outperforms the flow system both in the number of proteins modified (Figure 5) and the total FPOP coverage in those proteins. The number of FPOP modified proteins acquired in the platform incubator was approximately 1051, 2.2- fold more than those acquired in a typical experiment. Modifications were combined between two biological replicates for each experiment. Furthermore, PIXY provides higher throughput. 
To demonstrate the advantage of higher modification coverage across a protein, IC-FPOP modifications were localized on the peptide level and the extent of modification was quantified to distinguish differences in outcomes between the systems for actin, a 375 amino acid protein. In the flow system, two modified peptides were detected, providing limited structural information (Figure 6A). However, five modified peptides spanning the actin sequence were detected in the platform incubator. Tandem mass spectra indicate that residue Pro322 was both modified and detected in each experiment (Figure 6B). The five peptides modified in the platform incubator samples contained twelve modified residues, while only four residues were modified with the flow system (Figure 6C). The increase in oxidation coverage provides more structural information across the protein.

Espino et al. demonstrated the capacity of FPOP to be performed in vivo (IV-FPOP) within C. elegans, a worm model for human disease states ${ }^{17}$. While IV-FPOP is also performed via a flow system, the PIXY system was tested for compatibility with the worms. Approximately 10,000 worms were incubated in each well in the platform incubator at $20^{\circ} \mathrm{C}$. LC-MS/MS analysis revealed that 792 proteins were modified by IV-FPOP in the platform incubator compared to the 545 proteins modified with the flow system (Figure 7). These results demonstrate that in addition to $2 \mathrm{D}$ cell culture, this new methodology is also compatible with the study of other biological systems such as $C$. elegans. 


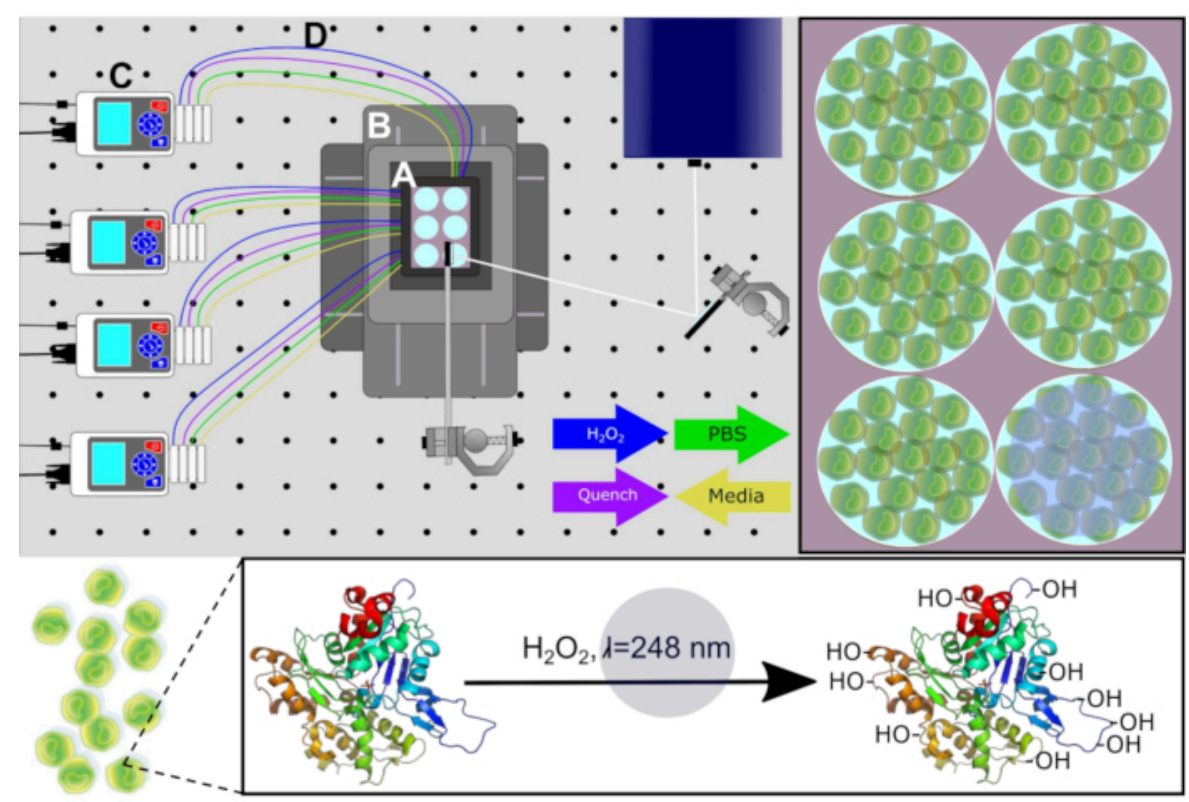

Figure 1. Schematic of PIXY System. System components: (A) stage-top incubator, (B) positioning system , (C) peristaltic pumps, and (D) perfusion lines. Cell culture media is removed from each well via pumps before $\mathrm{H}_{2} \mathrm{O}_{2}$ and quench solutions are infused at calculated timepoints. Laser path for irradiation showcased in white. Reprinted with permission from Johnson, D. T., Punshon-Smith, B., Espino, J. A., Gershenson, A., Jones, L. M., Implementing In-Cell Fast Photochemical Oxidation of Proteins in a Platform Incubator with a Movable XY Stage. Analytical Chemistry, 92(2), 1691-1696 2019. Copyright 2020 American Chemical Society. Please click here to view a larger version of this figure.

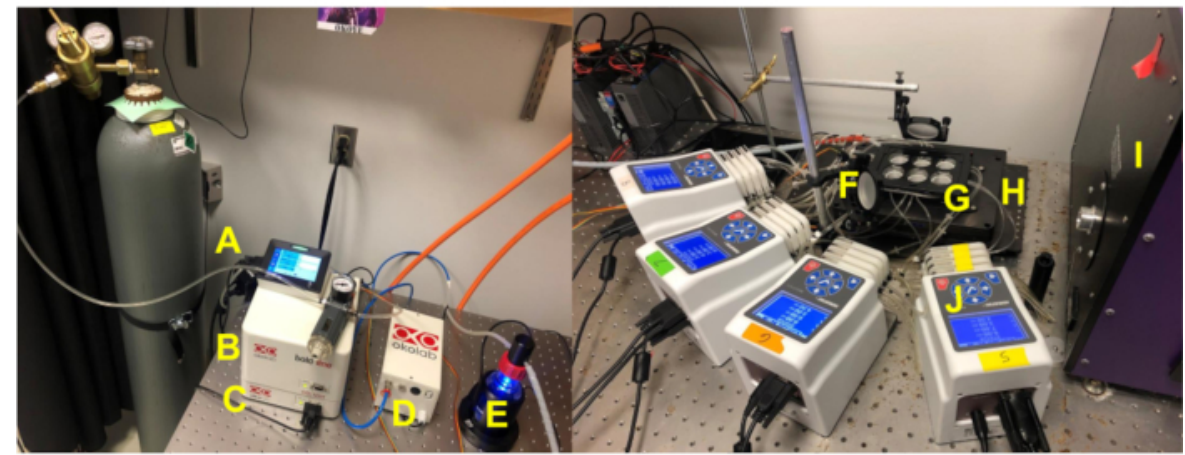

Figure 2. Fully Assembled PIXY system. (A) Touch monitoring system, (B) carbon dioxide unit, (C) temperature unit, (D) air pump, (E) humidifier, (F) optical mirrors, (G) platform incubator, (H) positioning stage, (I) 248nm KrF excimer laser, and (J) peristaltic pumps. Please click here to view a larger version of this figure. 


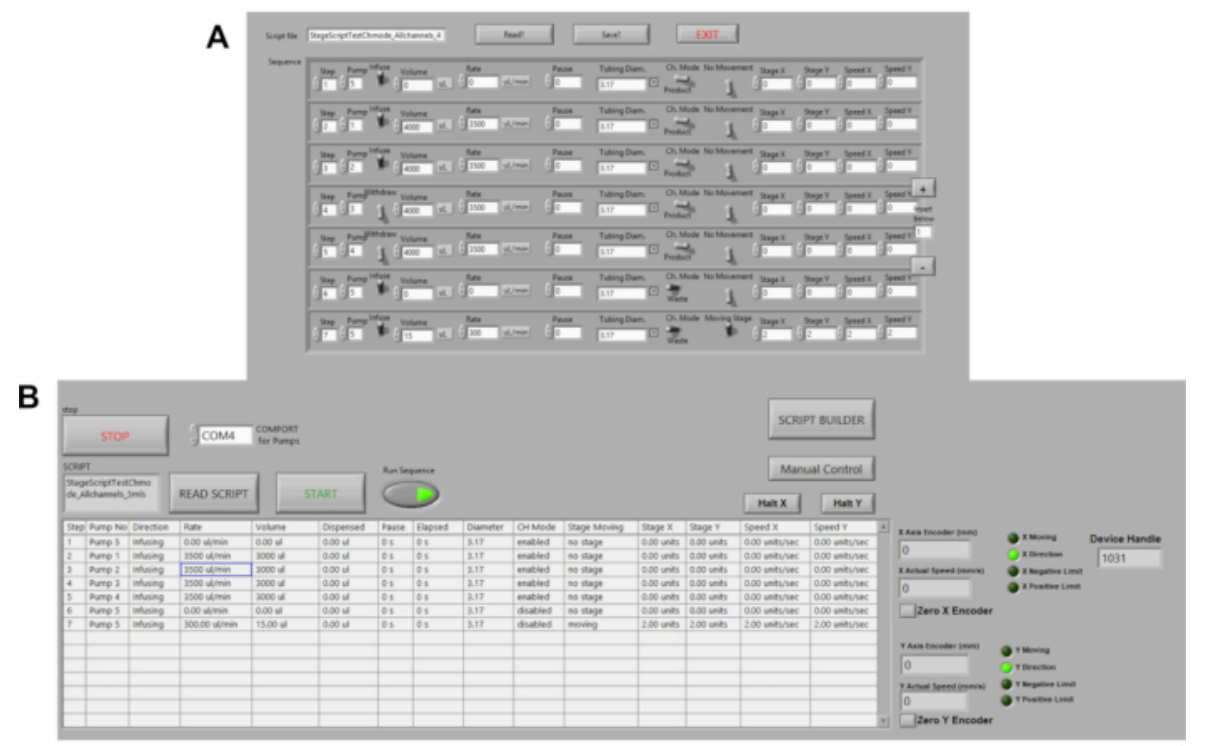

Figure 3. Automation of Peristaltic Pumps. (A) Example command script in LABVIEW. Command options include volume, flow rate, pauses, flow direction. Speed, stage distance, and location are currently being automated. (B) Script reader in LABVIEW. Here, command scripts are uploaded then Run Sequence and START are pressed to initiate pumps. Please click here to view a larger version of this figure. 
A

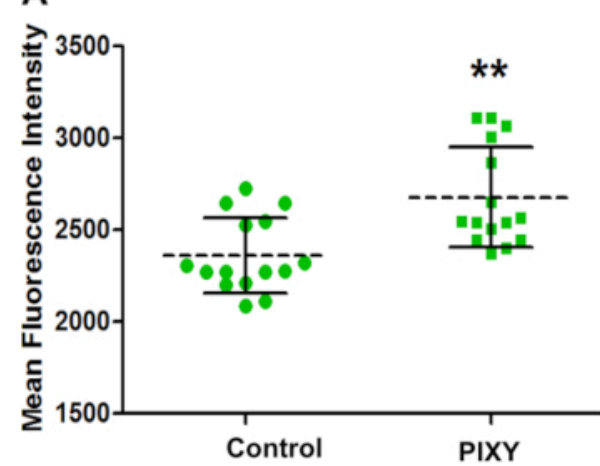

B

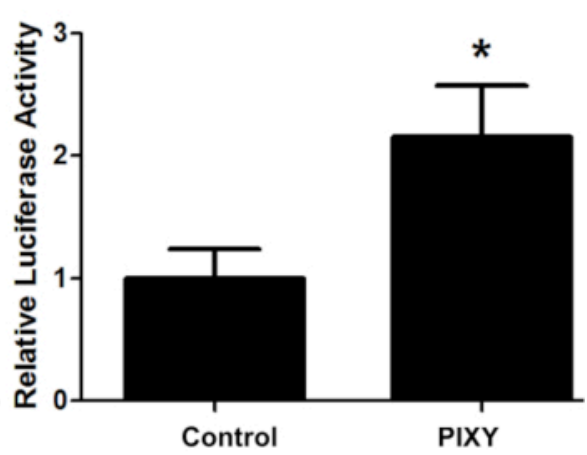

Figure 4. HEK cell transfection efficiency. (A) Mean fluorescent intensity of GCaMP2 transfection comparison between standard incubator (Control) and stage-top incubator (PIXY). Dots and squares represent each point in a well where a measure was taken. (B) Transfection efficiency quantitated and validated with a different vector plasmid, pRL-TK. P-value< 0.005. Reprinted with permission from Johnson, D. T., Punshon-Smith, B., Espino, J. A., Gershenson, A., Jones, L. M., Implementing In-Cell Fast Photochemical Oxidation of Proteins in a Platform Incubator with a Movable XY Stage. Analytical Chemistry, 92(2), 1691-1696 2019. Copyright 2020 American Chemical Society. Please click here to view a larger version of this figure. 


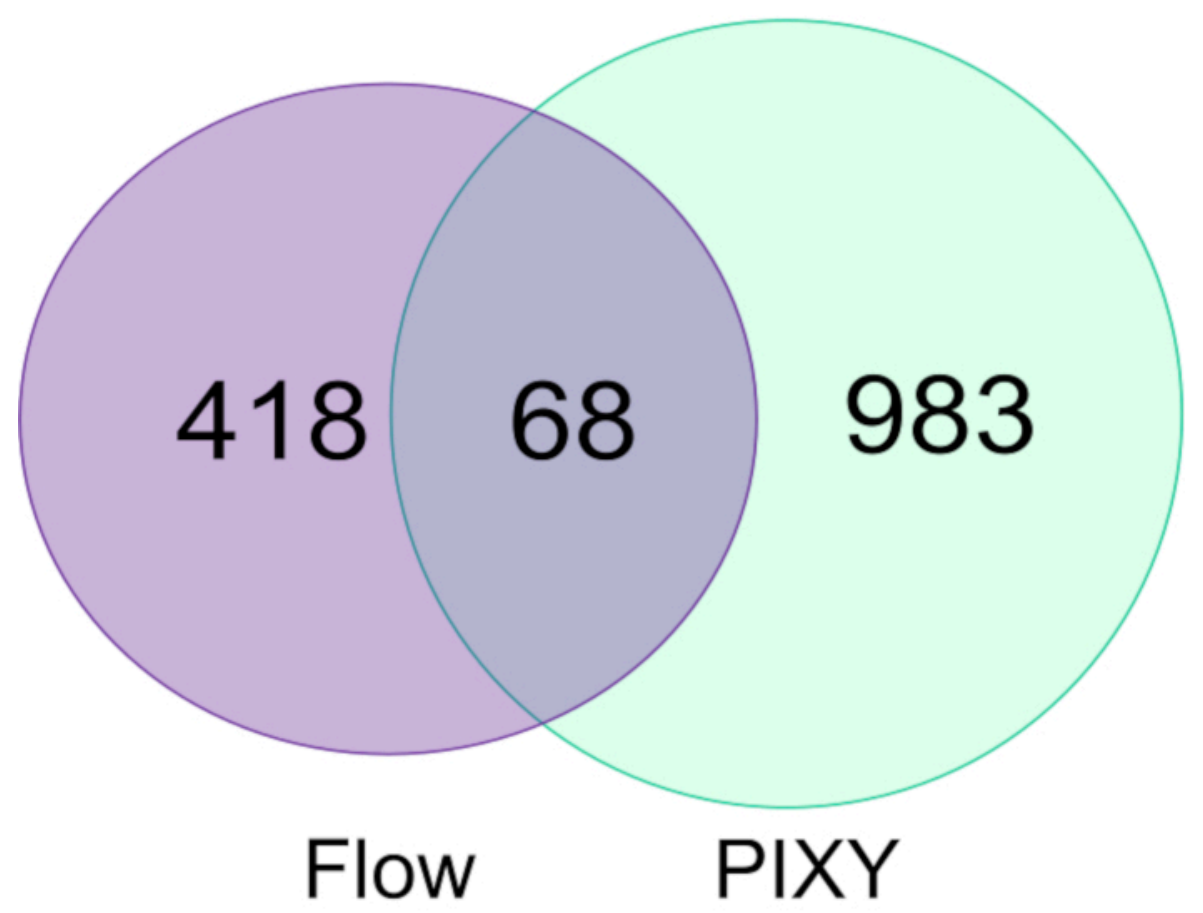

Figure 5. Comparison of proteins modified in the single cell flow system and PIXY. Venn diagram of proteins modified using in the flow system (purple) and in PIXY (green). Reprinted with permission from Johnson, D. T., Punshon-Smith, B., Espino, J. A., Gershenson, A., Jones, L. M., Implementing In-Cell Fast Photochemical Oxidation of Proteins in a Platform Incubator with a Movable XY Stage. Analytical Chemistry, 92(2), 1691-1696 2019. Copyright 2020 American Chemical Society. Please click here to view a larger version of this figure. 

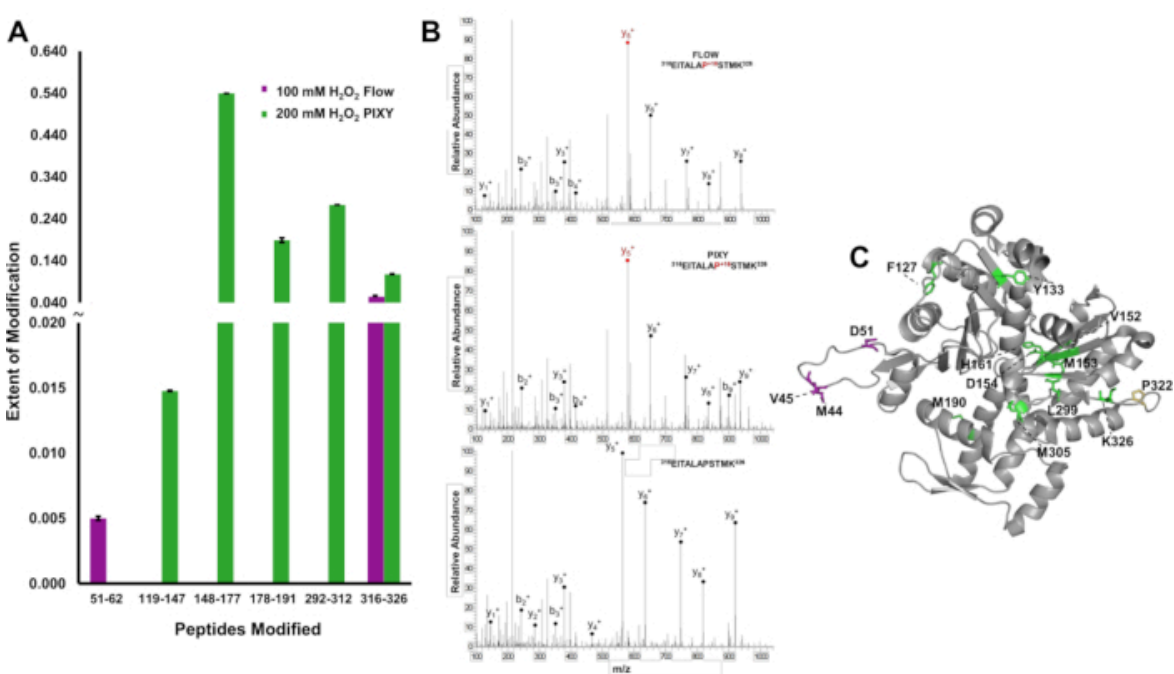

Figure 6. Localization of IC-FPOP modifications. Comparison of IC-FPOP modifications between systems. (A) Bar graph of oxidatively modified peptides within actin from the flow system (purple) vs platform incubator (green). (B) Tandem MS spectra of actin (peptide 316-326) with modified proline in both systems and unmodified actin peptide (C) FPOP modified residues of actin (PDB: 6ZXJ, chain A 11 modified residues in platform incubator (green), 3 modified residues in the flow system (purple), 1 overlapping modified residue (yellow). Reprinted with permission from Johnson, D. T., Punshon-Smith, B., Espino, J. A., Gershenson, A., Jones, L. M., Implementing In-Cell Fast Photochemical Oxidation of Proteins in a Platform Incubator with a Movable XY Stage. Analytical Chemistry, 92(2), 1691-1696 2019. Copyright 2020 American Chemical Society. Please click here to view a larger version of this figure. 


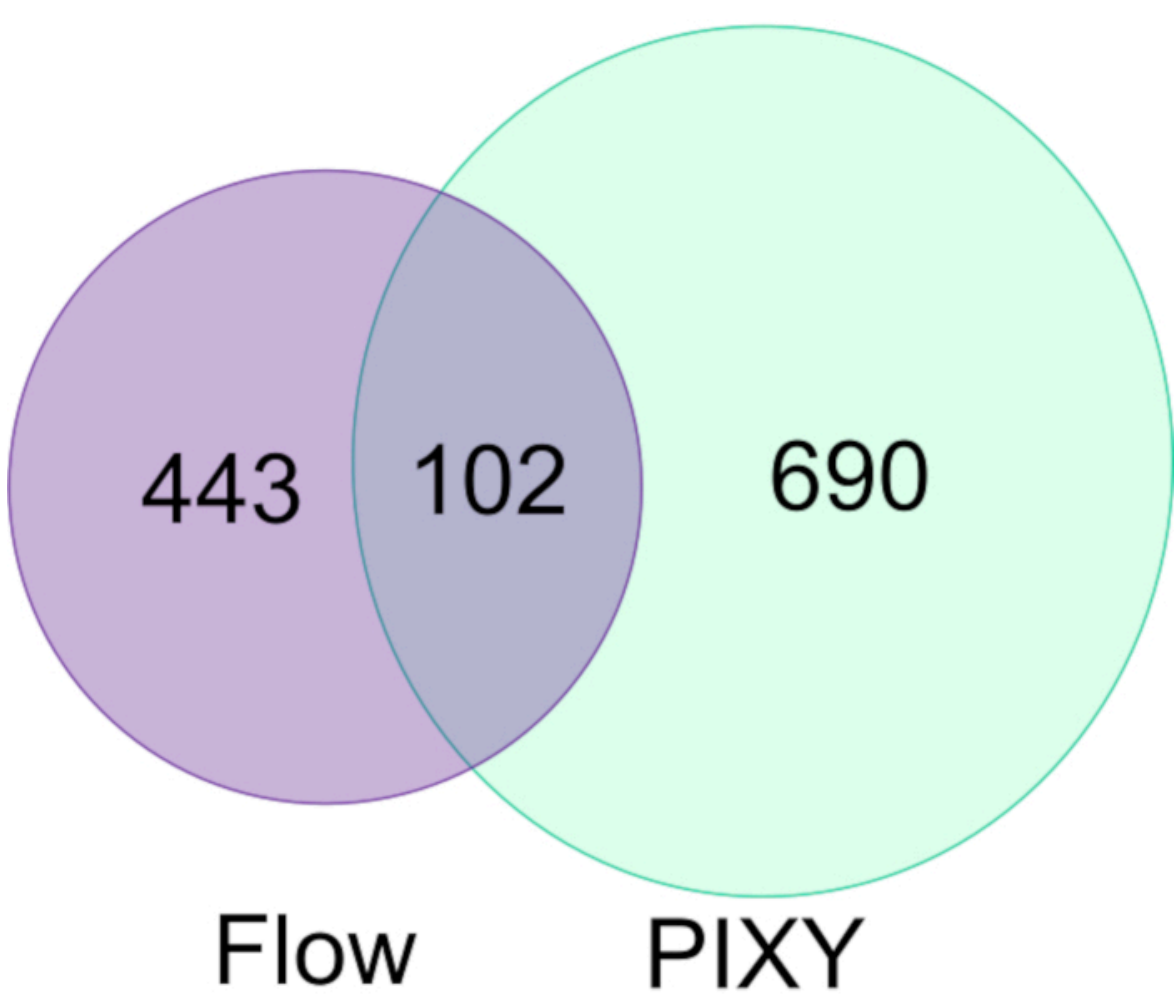

Figure 7. Comparison of FPOP modified proteins in C. elegans by flow vs PIXY. There is a 1.5 -fold increase in oxidatively modified proteins using PIXY when compared to the flow system. Reprinted with permission from Johnson, D. T., Punshon-Smith, B., Espino, J. A., Gershenson, A., Jones, L. M., Implementing In-Cell Fast Photochemical Oxidation of Proteins in a Platform Incubator with a Movable XY Stage. Analytical Chemistry, 92(2), 1691-1696 2019. Copyright 2020 American Chemical Society. Please click here to view a larger version of this figure.

Table 1. Workflow modification distribution and mass shifts (Da). Please click here to download this table.

\section{Discussion}

Proteins perform much of the work in living cells. Given this importance, detailed data on protein function and higher order structure (HOS) in the cellular environment is needed to deepen understanding of the intricacies in larger complexes and enzymatic reactions in cells as opposed to purified systems. To do this, a hydroxyl radical protein foot printing (HRFP) method was adopted entitled In-Cell
Fast Photochemical Oxidation of Proteins (IC-FPOP). Most FPOP studies have been done in vitro in relatively pure protein systems, which markedly contrasts with the crowded molecular environment which affects binding interactions and protein conformational dynamics. As a result, there is a chasm between the findings from in vitro experiments ${ }^{18}$ and those that would be obtained in an actual cellular environment. To bridge the gap between the idealized conditions of an in vitro FPOP experiment and the complex nature of the cell, a new automated six-well plate-based in cell-FPOP platform has been developed. This novel FPOP technology is capable of 
identifying and characterizing these molecular species and tracing their dynamic molecular interactions in both healthy and diseased states. This new platform is called Platform Incubator with Movable XY stage (PIXY).

FPOP has been successfully used to characterize the structural information within the proteome. However, every biological technique has certain limitations that require further improvement. Specific reagents are required during laser photolysis and to efficiently quench unreacted hydroxyl radicals. Separation of digested peptides can require large amounts of time to maximize structural information.This wealth of information can also require extensive quantitation during post-MS data analysis ${ }^{1}$. The platform incubator, including the peripheral machinery needed for cell culture and IC-FPOP at the laser platform, comes with a large cost that may not be feasible for some labs. As progress continues to be made, robust software and analysis tools should advance the technique further; some of which is showcased in this study. Current studies in this platform incubator have been performed on HEK293T cells and in C. elegans. The IC-FPOP method has been shown to be compatible with a wide variety of cell lines including Chinese hamster ovary $(\mathrm{CHO})$, Vero, MCF-7, and MCF10-A cells ${ }^{19}$. Since the general IC-FPOP method is translatable to this static platform, these cell lines should be amenable for study using PIXY as well.

IC-FPOP utilizes $\mathrm{H}_{2} \mathrm{O}_{2}$ to oxidatively modify solvent accessible side chains of amino acids, to then further discern protein interactions, structure, and metabolic effects within viable cells which is significant in providing biological context. It is essential before an IC-FPOP experiment to confirm that the cells are viable after $\mathrm{H}_{2} \mathrm{O}_{2}$ addition. Cell viability studies demonstrated that the cells were viable in the presence of $\mathrm{H}_{2} \mathrm{O}_{2}$ concentrations up to $200 \mathrm{mM}^{13}$. It is also important to make sure $\mathrm{H}_{2} \mathrm{O}_{2}$ is infused at a final concentration of $200 \mathrm{mM}$ directly on cells after media is removed. Failure to completely remove cell culture media will cause varying concentrations of $\mathrm{H}_{2} \mathrm{O}_{2}$. Compared to standard conditions, increasing the incubation time to 10 seconds along with increasing the $\mathrm{H}_{2} \mathrm{O}_{2}$ concentration led to a higher number of proteins modified by IC-FPOP in the platform incubator. It is imperative to prime peristaltic pumps before use to ensure pumps are working properly and liquid is being dispersed. Failure to do so may cause air bubbles in the tubing, insufficient volume of $\mathrm{H}_{2} \mathrm{O}_{2}$ to immerse cells, and/or insufficient volume of quench solution.

Another issue that may arise is unwanted delays in the system. An example of this is the process of verifying received commands for the pump systems which adds significant delays on the order of 1000 or more milliseconds using the integration software. This problem can be fixed by minimizing the communication with the pumps during the experiment and using pre-set commands ahead of time as much as possible.

In the future, the goal for PIXY is producing a fully automated and integrated system. In addition to the peristaltic pumps, the triggering of the laser pulse will be automated. A new positioning system will also be utilized for the rapid movement of the platform incubator to enhance speed and accuracy. All components of the system will continue to be programmed using the integration software to further increase throughput.

\section{Disclosures}

The authors declare no competing financial interest.

The research presented herein is associated with the below referenced patent application:

U.S. Non-Provisional Patent Application Number: 17/042,565 Title: "Device and Method for Determining Protein Folding" UMB Docket Number: LJ-2018-104 UMass Ref: UMA 18-059. 


\section{Acknowledgments}

This work was supported by a grant from the NIH R01 GM128983-01.

\section{References}

1. Johnson, D. T., Di Stefano, L. H., Jones, L. M. Fast photochemical oxidation of proteins (FPOP): A powerful mass spectrometry based structural proteomics tool. Journal of Biological Chemistry. 294 (32), 11969-11979 (2019).

2. Liu, X. R., Zhang, M. M., Gross, M. L. Mass Spectrometry-Based Protein Footprinting for Higher-Order Structure Analysis: Fundamentals and Applications. Chemical Reviews. 120 (10), 4355-4454 (2019).

3. Li, J., Chen, G. The use of fast photochemical oxidation of proteins coupled with mass spectrometry in protein therapeutics discovery and development. Drug Discovery Today. 24 (3), 829-883 (2019).

4. Zhao, B. et al. Covalent Labeling with an $\alpha, \beta-$ Unsaturated Carbonyl Scaffold for Studying Protein Structure and Interactions by Mass Spectrometry. Analytical Chemistry. 92 (9), 6637-6644 (2020).

5. Hambly, D. M., Gross, M. L. Laser Flash Photolysis of Hydrogen Peroxide to Oxidize Protein SolventAccessible Residues on the Microsecond Timescale. Journal of the American Society for Mass Spectrometry. 16 (12), 2057-2063 (2005).

6. Li, K. S., Shi, L., Gross, M. L. Mass Spectrometry-Based Fast Photochemical Oxidation of Proteins (FPOP) for Higher Order Structure Characterization. Accounts of Chemical Research. 51 (3), 736-744 (2018).
7. Chea, E. E., Jones, L. M. Modifications generated by fast photochemical oxidation of proteins reflect the native conformations of proteins. Protein Science. 27 (6), 1047-1056 (2018).

8. Hambly, D., Gross, M. Laser flash photochemical oxidation to locate heme binding and conformational changes in myoglobin. International Journal of Mass Spectrometry. 259 (1), 124-129 (2007).

9. Yan, Y. Fast photochemical oxidation of proteins (FPOP) maps the epitope of EGFR binding to adnectin. Journal of the American Society for Mass Spectrometry. 25 (12), 2084-2092 (2014).

10. Zhang, Y., Wecksler, A. T., Molina, P., Deperalta, G., Gross, M. L. Mapping the Binding Interface of VEGF and a Monoclonal Antibody Fab-1 Fragment with Fast Photochemical Oxidation of Proteins (FPOP) and Mass Spectrometry. Journal of the American Society for Mass Spectrometry. 28 (5), 850-858 (2017).

11. Espino, J. A., Mali, V. S., Jones, L. M. In Cell Footprinting Coupled with Mass Spectrometry for the Structural Analysis of Proteins in Live Cells. Analytical Chemistry. 87 (15), 7971-7978 (2015).

12. Rinas, A., Mali, V. S., Espino, J. A., Jones, L. M. Development of a Microflow System for In-Cell Footprinting Coupled with Mass Spectrometry. Analytical Chemistry. 88 (20), 10052-10058 (2016).

13. Johnson, D. T., Punshon-Smith, B., Espino, J. A., Gershenson, A., Jones, L. M. Implementing InCell Fast Photochemical Oxidation of Proteins in a Platform Incubator with a Movable XY Stage. Analytical Chemistry. 92 (2), 1691-1696 (2019). 
14. Bangel-Ruland, N. et al. Cystic fibrosis transmembrane conductance regulator-mRNA delivery: a novel alternative for cystic fibrosis gene therapy. The Journal of Gene Medicine. 15 (11-12), 414-426 (2013).

15. Gau, B. C., Chen, H., Zhang, Y., Gross, M. L. Sulfate Radical Anion as a New Reagent for Fast Photochemical Oxidation of Proteins. Analytical Chemistry. 82 (18), 7821-7827 (2010).

16. Xu, G. C., M. Hydroxyl Radical-Mediated Modification of Proteins as Probes for StructuralProteomics. Chemical Reviews. American Chemical Society. 107 (8), 3514-3543 (2007).

17. Espino, J. A., Jones, L. M. Illuminating Biological Interactions with In Vivo Protein Footprinting. Analytical Chemistry. 9(10), 6577.-6584 (2019).

18. Gau, B. C., Sharp, J. S., Rempel, D. L., Gross, M. L. Fast Photochemical Oxidation of Protein Footprints Faster than Protein Unfolding. Analytical Chemistry. 81 (16), 6563-6571 (2009).

19. Kaur, U., Johnson, D. T., Jones, L. M. Validation of the Applicability of In-Cell Fast Photochemical Oxidation of Proteins across Multiple Eukaryotic Cell Lines. Journal of the American Society for Mass Spectrometry. 31 (7), 1372-1379 (2020). 